\title{
Broad-Host-Range cre-lox System for Antibiotic Marker Recycling in Gram-Negative Bacteria
}

BioTechniques 33:1062-1067 (November 2002)

\section{Christopher J. Marx and Mary E. Lidstrom University of Washington, Seattle, WA, USA}

\begin{abstract}
Complete genome sequences are now available for many bacterial species that lack sophisticated genetic tools. We describe the development of a broad-host-range crelox system that allows antibiotic marker recycling in a variety of Gram-negative bacteria. This system consists of an allelic exchange vector bearing a kanamycin cassette flanked by loxP sites and a tetracyclineresistant IncP plasmid that provides expression of the Cre recombinase. We demonstrate this system by generating unmarked deletions of genes in two different bacteria, Methylobacterium extorquens AM1 and Burkholderia fungorum LB400. This new antibiotic marker recycling system offers the possibility of creating unmarked mutants in a wide variety of Gram-negative bacteria. Furthermore, marker recycling allows the generation of strains bearing multiple genetic manipulations in organisms for which few antibiotic markers are currently available.
\end{abstract}

\section{INTRODUCTION}

The availability of complete genome sequences for a wide variety of microbial species has revolutionized microbiology. Although bioinformatics tools permit phylogenetic classification of the majority of the putative gene products into conserved classes (16), the annotation of function based on sequence alone is still, by its very nature, a hypothesis. Experimentation is required to test and refine these hypotheses. For many of these organisms, however, the genetic toolkit available is limited, at best. One key genetic hurdle slowing progress is the lack of facile tools to generate unmarked mutant strains in a wide variety of organisms. The generation of unmarked mutants allows for multiple genetic manipulations of organisms for which few antibiotic markers exist. In recent years, a growing number of systems for antibiotic marker recycling in non-enteric bacteria have been reported that utilize a variety of site-specific recombination systems and antibiotic markers (5). These include the utilization of the RP4 multimer resolution system (8) and both the yeast Flp/FRT (7) and P1 phage cre/lox (2) site-specific recombination systems. This paper describes the development of a simple broad-host-range antibiotic marker recycling system based on crelox methodology. Cre recombinase is a site-specific recombinase from the $\mathrm{P} 1$ phage that catalyzes in vivo excision of DNA regions flanked by co-directional lox $P$ recognition sites (12). The system we describe consists of a mobilizable allelic exchange vector with a loxPflanked antibiotic resistance cassette, pCM184 or pCM351, and an IncP plas- mid that expresses the Cre recombinase, pCM157 or pCM158. We demonstrate the broad utility of this system by generating unmarked mutant strains of two phylogenetically distinct Gramnegative bacteria, Methylobacterium extorquens AM1 (an $\alpha$-proteobacterium) and Burkholderia fungorum LB400 (a $\beta$-proteobacterium).

\section{MATERIALS AND METHODS}

\section{Media and Growth Conditions}

M. extorquens AM1 (11) and B. fungorum LB400 (3) strains were grown on a minimal salts medium (1) containing carbon sources at the following levels, $0.2 \%$ citrate, $0.5 \%$ (v/v) methanol, and $0.4 \%$ (w/v) succinate. E. coli strains were grown on LB medium (14) (strains and plasmids are described in Table 1). Antibiotics were added at the following final concentrations, unless noted: 50 $\mu \mathrm{g} / \mathrm{mL}$ ampicillin, $10 \mu \mathrm{g} / \mathrm{mL}$ chloramphenicol, $50 \mu \mathrm{g} / \mathrm{mL}$ (for $E$. coli and $M$. extorquens AM1), or $20 \mu \mathrm{g} / \mathrm{mL}$ (for $B$. fungorum LB400) kanamycin, $50 \mu \mathrm{g} /$ $\mathrm{mL}$ rifamycin, $35 \mu \mathrm{g} / \mathrm{mL}$ streptomycin, and $10 \mu \mathrm{g} / \mathrm{mL}$ tetracycline. Chemicals were obtained from Sigma (St. Louis, MO, USA). Nutrient agar and Bactoagar were obtained from Difco (Detroit, MI, USA). Conjugation was performed as described previously (4).

\section{Construction of a Broad-Host- Range cre-lox System for Antibiotic Marker Recycling}

Two allelic exchange vectors, pCM184 and pCM351 (Figure 1), were created by inserting loxP-bounded anti- 
biotic resistance cassettes into a variant of the mobilizable suicide plasmid, pAYC61 (4). The 1.3-kb HincII fragment bearing the kanamycin resistance cassette from pUC4K (17) was inserted into pLox1 (12), which had been cut with XbaI and blunted, to create pCM161. To introduce convenient multiple cloning sites, the loxP-bounded kanamycin cassette of pCM161 was amplified with following primer pair, CM-ufkMCS, 5'-TGACGTCTAGATCTGAATTCAGCTGTACAATTGGTACCATGGATGCATATGGCGGCCGCA-3', and CM-dfkMCS, 5'-GACTAGTGAGCTCACCGGTTAACACGCGTACGTAGGGCCCGCGGTATCGATAAGCTGGATCC-3'. The resulting 1.4-kb PCR product was purified and cloned into pCR2.1 (Invitrogen, Carls- bad, CA, USA) to create pCM183. To preserve useful cloning sites, pAYC61 was cut with EcoRI and SmaI, blunted using T4 DNA polymerase, and selfligated to produce pCM182. Finally, the 1.4-kb AatII-SpeI fragment from pCM183 containing the loxP-flanked kanamycin cassette was ligated between the AatII and XbaI sites of pCM182 to create pCM184 (Gen-

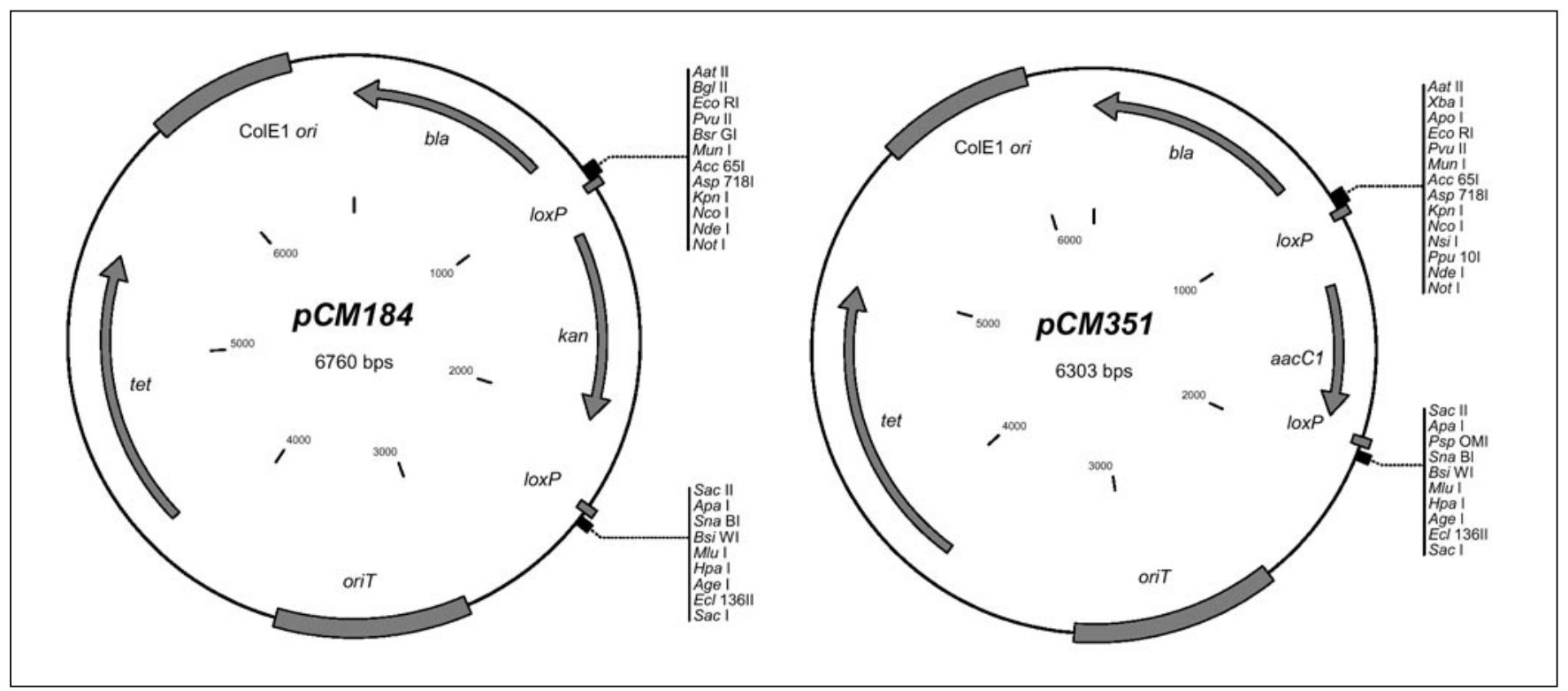

Figure 1. Plasmid map of the allelic exchange vectors pCM184 and pCM351. Antibiotic resistances are encoded by bla (ampicillin), aaaC1 (gentamycin), kan (kanamycin), and tet (tetracycline). The boxes indicate the pair of loxP sites (loxP), the IncP origin of tranfer (oriT), and the ColE1 origin of replication (ColE1 ori). The unique restriction sites present in the multiple cloning sites are indicated.
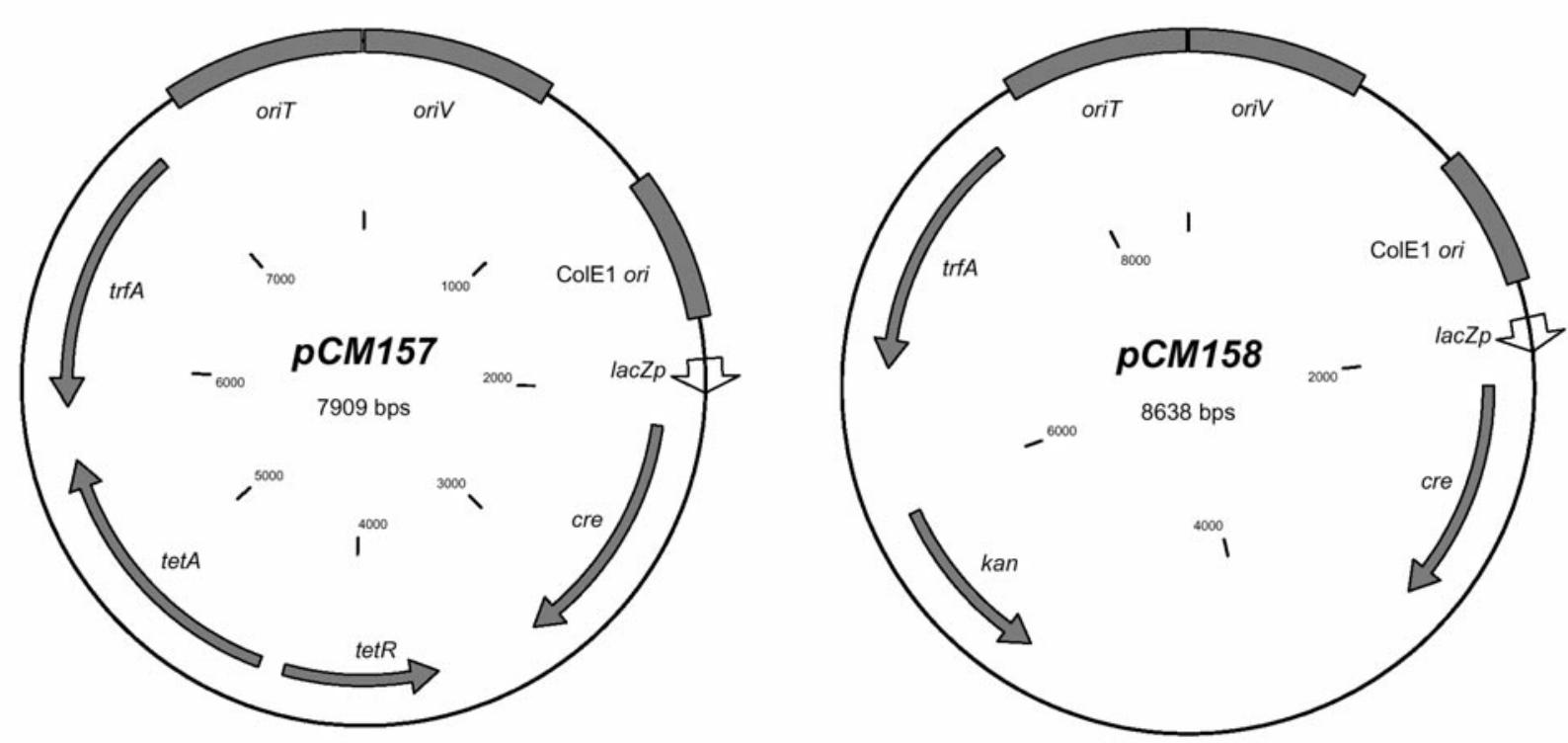

Figure 2. Plasmid map of the cre expression plasmids pCM157 and pCM158. The E. coli lacZp drives expression of Cre recombinase. These two plasmids differ only in the antibiotic resistance genes present, tetAR (tetracycline) or kan (kanamycin). The boxes indicate the IncP origin of replication (oriV), the ColE1 origin of replication (ColE1 ori), and the IncP origin of tranfer (oriT). 


\section{Research Report}

Bank $^{\circledR}$ accession no. AY093429). A gentamycin-resistance conferring version, pCM351, was also generated. The loxP-flanked gentamycin-resistance cassette (encoded by aaaC1) was amplified from pLoxGen4 (12), using CM-ufkMCS and CM-dfkMCS, and cloned into pCR2.1 (Invitrogen) to produce pCM350. The 1-kb AatII/SacI fragment from pCM350 was cloned between the AatII and SacI sites of pCM184 to generate pCM351 (GenBank accession no. AY093430).

Two broad-host-range cre expression vectors, pCM157 and pCM158 (Figure 2), were created based on a pair of small, mobilizable IncP plasmids (10). The 1.1-kb XbaI-EcoRI fragment from pJW168 (19) was cloned between the $X b a \mathrm{I}$ and EcoRI sites of pCM62 (10) to generate the tetracycline-resistance conferring cre expression plasmid pCM157. A kanamycin-resistant version, pCM158, was generated by cloning the same $X b a \mathrm{I}-E c o$ RI fragment from pJW168 between the XbaI and EcoRI sites of pCM66 (10). These plasmids contain cre behind the E. coli lac promoter. In $M$. extorquens AM1, this promoter provides only low constitutive activity (10). Despite this low expression, the majority of cells obtained from the first passage onto plates lacking kanamycin are already kanamycin sensitive (data not shown).

\section{Generation of a $\Delta$ fae Mutant of $M$. extorquens AM1}

M. extorquens AM1 mutants defective for fae (encodes formaldehyde-activating enzyme) (18) were generated using pCM184 (Figure 2). The regions immediately flanking fae were amplified by PCR using the following primer pairs: CM-Dfae1, 5'-CGGGTTTCGTGACCTGTTC-3', and CM-Dfae2, 5' GTTATGCGGCCGCCATCTGCATGGAAGCCATCCTTGTTTGC-3'; and CM-Dfae3, 5'-GCTTATCGATACCGTCGACCTCGAGGCAGTCCTGGGCAGA-3', and CM-Dfae4, 5'-CGGGCATCGAGCGTTTCAC-3'. The purified PCR products for fae-upstream and fae-downstream were cloned into pCR2.1 to produce pCM195 and

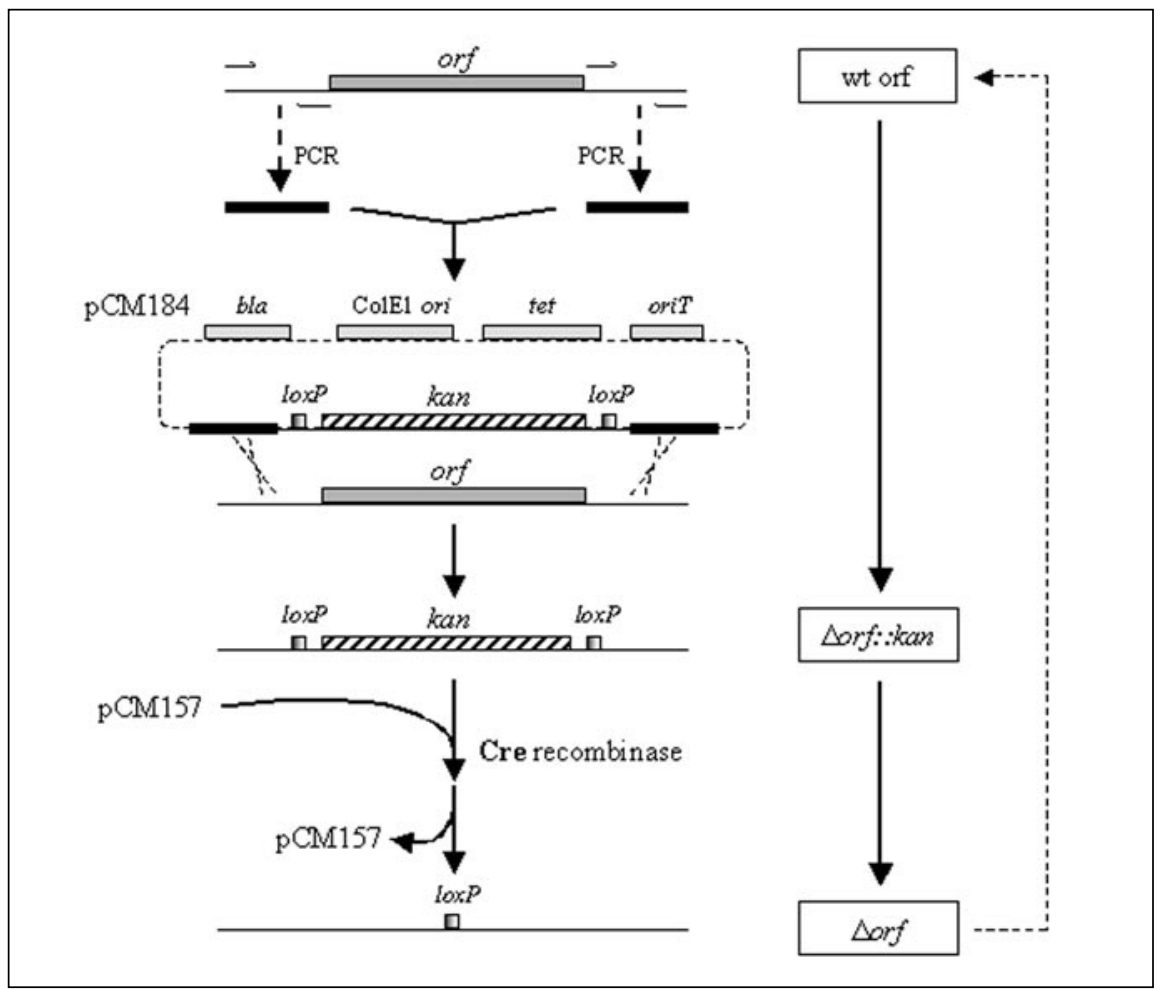

Figure 3. Strategy for antibiotic marker recycling. DNA flanks upstream and downstream of the target gene are amplified by PCR and cloned into pCM184. Allelic exchange leads to a kan insertion mutant, which can then be unmarked through the introduction of the cre expression plasmid pCM157. The process can then be repeated with a second target gene to generate a strain bearing multiple genetic manipulations. 
Table 1. Bacterial Strains and Plasmids Used in This Study

\begin{tabular}{|c|c|c|}
\hline Strain/Plasmid & Relevant Properties & Reference \\
\hline \multicolumn{3}{|l|}{ Strains } \\
\hline B. fungorum LB400 & Polychlorinated biphenyls-degrading isolate & 3 \\
\hline СM363К.1 & $\Delta$ flhA::kan B. fungorum LB400 & This study \\
\hline СM363.1 & $\Delta$ flhA B. fungorum LB400 & This study \\
\hline E. coliS17-1 & C600::RP-4 2-(Tc::Mu) (Kn::Tn7) thi pro hsdR hsdM+ recA & 15 \\
\hline M. extorquens AM1 & Rifr derivative & 11 \\
\hline CM198K.1 & $\Delta$ fae::kan M. extorquens AM1 & This study \\
\hline CM198.1 & $\Delta$ fae M. extorquens AM1 & This study \\
\hline \multicolumn{3}{|l|}{ Plasmids } \\
\hline pAYC61 & $A p^{r}, T c^{r} ;$ mobilizable allelic exchange vector & 4 \\
\hline pCM62 & Tcr; broad-host-range cloning vector & 10 \\
\hline pCM66 & $\mathrm{Kn}^{\mathrm{r}}$; broad-host-range cloning vector & 10 \\
\hline pCM157 & Tc'; pCM62 with cre from pJW168; cre expression vector & This study \\
\hline pCM158 & $\mathrm{Kn}$; pCM66 with cre from pJW168; cre expression vector & This study \\
\hline pCM161 & $A p^{r}, K^{r} ;$ pLox1 with kan cassette from pUC4K & This study \\
\hline pCM182 & $A p^{r}, T^{r} ;$ pAYC61 cut with EcoRI and Smal, blunted, and self-ligated & This study \\
\hline pCM183 & $\mathrm{Ap}^{\mathrm{r}} ; \mathrm{Kn}^{\mathrm{r}} ; \mathrm{pCR} 2.1$ with kan cassette amplified from $\mathrm{pCM} 161$ & This study \\
\hline pCM184 & $\mathrm{Ap}^{r}, \mathrm{Kn}^{r}, \mathrm{Tc}^{\mathrm{r}} ; \mathrm{pCM} 182$ with kan from $\mathrm{pCM} 183$; allelic exchange vector & This study \\
\hline pCM195 & $\mathrm{Apr}, \mathrm{Kn} ;$; pCR2.1 with fae upstream flank & This study \\
\hline pCM196 & $A p^{r}, K^{r} ; p C R 2.1$ with fae downstream flank & This study \\
\hline pCM197 & $\mathrm{Ap}^{r}, \mathrm{Kn}^{r}, \mathrm{Tc}^{\mathrm{r}} ; \mathrm{pCM} 184$ with fae upstream flank & This study \\
\hline pCM198 & $\mathrm{Ap}^{r}, \mathrm{Kn}^{r}, \mathrm{Tc}^{r} ; \mathrm{pCM} 197$ with fae downstream flank; donor for $\Delta$ fae::kan & This study \\
\hline pCM350 & $\mathrm{Ap}^{\mathrm{r}} ; \mathrm{Gm}^{\mathrm{r}}, \mathrm{Kn}^{\mathrm{r}} ; \mathrm{pCR} 2.1$ with aaaC1 cassette amplified from pLoxGen4 & This study \\
\hline pCM351 & $\mathrm{Ap}^{r}, \mathrm{Gm} r, \mathrm{Tc}$; $\mathrm{pCM} 184$ with aaaC1 from pCM350; allelic exchange vector & This study \\
\hline pCM360 & $\mathrm{Ap}^{\mathrm{r}}, \mathrm{Kn} \mathrm{K}^{\mathrm{r}} ; \mathrm{pCR} 2.1$ with flhA upstream flank & This study \\
\hline рСМ361 & $\mathrm{Ap}^{r}, \mathrm{Kn}{ }^{r} ; \mathrm{pCR} 2.1$ with flhA downstream flank & This study \\
\hline рСМ362 & $A p^{r}, \mathrm{Kn}^{r}, \mathrm{~T} \mathrm{c}^{r} ; \mathrm{pCM} 184$ with flhA upstream flank & This study \\
\hline рСМ363 & $\mathrm{Ap}^{r}, \mathrm{Kn}{ }^{\mathrm{r}}, \mathrm{Tc} \mathrm{c}^{\mathrm{r}}$; $\mathrm{pCM} 62$ with flhA downstream flank; donor for $\Delta f l h A:: k a n$ & This study \\
\hline pCR2.1 & $\mathrm{Ap}^{r}, \mathrm{Km} r ; \mathrm{PCR}$ cloning vector & Invitrogen \\
\hline pJW168 & Apr $;$ cre expression plasmid & 19 \\
\hline pLox1 & Apr; mobilizable suicide vector with loxP sites & 12 \\
\hline pLoxGen4 & $A p^{r}, \mathrm{Gm}^{r} ;$ pLox 1 with aaaC1 cloned between loxP sites & 12 \\
\hline pRK2073 & Smr helper plasmid supplying IncP tra functions & 6 \\
\hline pUC4K & $A p^{r}, K_{m}^{r} ;$ vector with kan cassette & 17 \\
\hline
\end{tabular}

Antibiotic resistances are indicated as follows, Ap (ampicillin), Gm (gentamycin), Kn (kanamycin), Rif (rifamycin), Sm (streptomycin), and Tc (tetracycline).

pCM196, respectively. The $0.6-\mathrm{kb}$ EcoRI-NotI fragment from pCM195 was introduced between the EcoRI and NotI sites of pCM184 to produce pCM197. Subsequently, the $0.6-\mathrm{kb}$ ApaI-SacI fragment from pCM196 was ligated between the ApaI and SacI sites of pCM197 to produce pCM198.
A $\Delta$ fae::kan mutant of $M$. extorquens AM1 was generated by introducing pCM198 by conjugation from E. coli S17-1 (15). Kanamycin-resistant transconjugants obtained on succinate medium containing rifamycin were screened for tetracycline sensitivity to identify potential null mutants. To date, our laboratory has generated more than 30 different null mutant strains utilizing this system, and the frequency of double-crossover events has varied from $5 \%$ to $80 \%$ (unpublished data). One such $\Delta$ fae::kan mutant, CM198K.1, was chosen for further study. The plasmid pCM157 was introduced by conju- 
gation into CM198K.1 using the helper plasmid pRK2073 (6). Tetracycline-resistant strains were streaked for purity until the resulting strain produced only kanamycin-sensitive colonies (generally only two transfers). Subsequently, pCM157 was cured from the strain by two successive transfers on medium lacking tetracycline to produce the $\Delta f a e$ strain CM198.1. Analytical PCR was performed with wild-type $M$. extorquens AM1, CM198K.1, and CM198.1 for the confirmation of allelic exchange and the subsequent deletion of the kanamycin cassette (data not shown). Where examined, the sequence of the analytical PCR product indicated faithful recombination between the loxP sites (data not shown).

\section{Generation of a $\Delta f l h A$ Mutant of $B$. fungorum LB400}

B. fungorum LB400 mutants defective for flhA (predicted to encode a NAD- and glutathione-dependent formaldehyde dehydrogenase) (13) were generated using pCM184, as described earlier with $M$. extorquens AM1. The regions flanking flhA were amplified by PCR using the following primer pairs: CM-BfflhAuf, 5'-GGTGACGGCATTGAAGCTG-3', and CM-BfflhAur, 5'-CATGCATCTTTGGTCTTCATCGTGAATG-3'; and CM-BfflhAdf, 5'-ACCGCGGTCGTGCTGTACTAATCC-3', and CM-BfflhAur, 5'-AGAGCTCGATACCGACCGATAGATCTC- ${ }^{\prime}$. The flhA upstream and downstream PCR products were cloned into pCR2.1 (Invitrogen) to produce pCM360 and pCM361, respectively. The $0.6-\mathrm{kb}$ SacII-SacI downstream fragment from pCM361 was introduced between the SacII and SacI sites of pCM184 to produce pCM362. Subsequently, the $0.5-\mathrm{kb}$ EcoRI-Nsi upstream fragment from pCM360 was ligated between the EcoRI and NsiI sites of pCM362 to produce pCM363.

A $\triangle$ flhA::kan mutant of B. fungorum LB400 was generated by introducing pCM363 by conjugation. Kanamycinresistant transconjugants were obtained on citrate medium containing chloramphenicol (wild-type B. fungorum LB400 was found to be naturally resistant below $10-20 \mu \mathrm{g} / \mathrm{mL}$ ). One tetracyclinesensitive strain representing a $\triangle$ flhA:: kan mutant, CM363K.1, was chosen for further study. The plasmid pCM157 was used as described earlier to produce the $\triangle$ flhA strain CM363.1. Analytical PCR was performed with wildtype B. fungorum LB400, CM363K.1, and CM363.1 for confirmation (data not shown).

The minimal inhibitory concentration of formaldehyde was determined by comparing the rate and extent of colony formation of wild-type $B$. fungorum LB400 to that of the flhA mutants CM363K.1 and CM363.1 on solid medium containing succinate as a growth substrate with various concentrations of formaldehyde. Formaldehyde was added to the plates immediately before the addition of the molten agar. Because an undetermined fraction of the formaldehyde will volatilize, the reported minimal inhibitory concentration of formaldehyde is a maximum value.

\section{RESULTS AND DISCUSSION}

To test the broad-host-range cre-lox antibiotic marker recycling system, unmarked mutants were generated in $M$. extorquens AM1 (an $\alpha$-proteobacterium) and $B$. fungorum LB400 (a $\beta$-proteobacterium). Analytical PCR confirmed replacement of each deleted gene with kan and the subsequent excision of kan to produce the unmarked deletion (data not shown). The $\Delta$ fae mutant of $M$. extorquens AM1 grew like the wild-type on succinate but failed to grow on methanol or medium containing succinate and methanol. This mutant phenotype is in agreement with previous observations with a fae::kan mutant (18). The CM198.1 $\Delta$ fae strain can serve as a convenient host for structure-function studies that require the expression of variant Fae proteins.

As a second demonstration of this broad-host-range antibiotic marker recycling system, a $\triangle f l h A$ mutant of $B$. fungorum LB400 was generated. In other bacteria, the flhA gene encodes a glutathione-dependent formaldehyde dehydrogenase (13). This enzyme is involved in formaldehyde detoxification in E. coli (9) and Paracoccus denitrificans (13) and is required for methylotrophic growth by the latter. The $\Delta f l h A$ strain CM363.1 was found to be 
somewhat more sensitive to the presence of formaldehyde during growth on citrate than wild-type $B$. fungorum LB400, with a minimal inhibitory concentration of $0.1 \mathrm{mM}$ compared to 0.2 $\mathrm{mM}$ for the wild-type. This finding demonstrates that the glutathione-dependent pathway is involved in formaldehyde detoxification across multiple branches of the proteobacteria.

In conclusion, this new broad-hostrange cre-lox antibiotic marker recycling system offers the possibility to create unmarked mutants in a wide variety of Gram-negative bacteria. Utilization of allelic exchange with counter-selection against integrants and an inherently unstable minimal IncP Cre expression plasmid obviates the need for successful negative selection in the target organism, a feature of some previously developed marker recycling systems (7). The use of PCR to generate flanks for gene replacement allows for the facile generation of precise deletion mutants (Figure 3), as well as truncations through the introduction of start or stop codons in the primers, as needed. Variants of this system can be readily developed to allow the construction of chromosomal transcriptional or translational fusions (T. Strovas, C.J. Marx, and M.E. Lidstrom, unpublished data). Marker recycling systems such as ours described here offer a substantial advantage over standard allelic exchange methods because it can be used iteratively to enable the generation of unmarked strains bearing multiple genetic modifications. Our laboratory has already used this system to generate an M. extorquens AM1 strain bearing four separate mutations (C.J. Marx, L. Chistoserdova, and M.E. Lidstrom, unpublished data). Finally, engineered strains generated with these tools are more acceptable for environmental release, owing to the absence of introduced antibiotic resistance markers.

\section{ACKNOWLEDGMENTS}

We would like to thank F. Baneyx for providing strains and L. Chistoserdova and to T. Strovas for sharing unpublished data. This work was funded by National Institutes of Health grant no. GM39646.

\section{REFERENCES}

1.Attwood, M.M. and W. Harder. 1972. A rapid and specific enrichment procedure for Hyphomicrobium spp. Antonie Van Leeuwenhoek 38:369-377.

2.Ayres, E.K., V.J. Thomson, G. Merino, D. Balderes, and D.H. Figurski. 1993. Precise deletions in large bacterial genomes by vectormediated excision (VEX). The trfA gene of promiscuous plasmid RK2 is essential for replication in several Gram-negative hosts. J. Mol. Biol. 230:174-185.

3.Bopp, L.H. 1986. Degradation of highly chlorinated PCBs by Pseudomonas strain LB400. J. Ind. Microbiol. 1:23-29.

4.Chistoserdov, A.Y., L.V. Chistoserdova, W.S. McIntire, and M.E. Lidstrom. 1994. Genetic organization of the mau gene cluster in Methylobacterium extorquens AM1: complete nucleotide sequence and generation and characteristics of mau mutants. J. Bacteriol. 176:4052-4065.

5.Davison, J. 2002. Genetic tools for pseudomonads, rhizobia, and other Gram-negative bacteria. BioTechniques 32:386-394.

6.Figurski, D.H. and D.R. Helinski. 1979. Replication of an origin-containing derivative of plasmid RK2 dependent on a plasmid function provided in trans. Proc. Natl. Acad. Sci. USA 76:1648-1652.

7.Hoang, T.T., R.R. Karkhoff-Schweizer, A.J. Kutchma, and H.P. Schweizer. 1998. A broad-host-range Flp-FRT recombination system for site-specific excision of chromosomally-located DNA sequences: application for isolation of unmarked Pseudomonas aeruginosa mutants. Gene 212:77-86.

8.Kristensen, C.S., L. Eberl, J.M. SanchezRomero, M. Givskov, S. Molin, and V. De Lorenzo. 1995. Site-specific deletions of chromosomally located DNA segments with the multimer resolution system of broad-hostrange plasmid RP4. J. Bacteriol. 177:52-58.

9.Kummerle, N., H.H. Feucht, and P.M. Kaulfers. 1996. Plasmid-mediated formaldehyde resistance in Escherichia coli: characterization of resistance gene. Antimicrob. Agents Chemother. 40:2276-2279.

10.Marx, C.J. and M.E. Lidstrom. 2001. Development of improved versatile broad-hostrange vectors for use in methylotrophs and other Gram-negative bacteria. Microbiology 147:2065-2075.

11.Nunn, D.N. and M.E. Lidstrom. 1986. Isolation and complementation analysis of 10 methanol oxidation mutant classes and identification of the methanol dehydrogenase structural gene of Methylobacterium sp. strain AM1. J. Bacteriol. 166:581-590.

12.Palmeros, B., J. Wild, W. Szybalski, S. Le Borgne, G. Hernandez-Chavez, G. Gosset, F. Valle, and F. Bolivar. 2000. A family of removable cassettes designed to obtain antibiotic-resistance-free genomic modifications of Escherichia coli and other bacteria. Gene 247:255-264.

13.Ras, J., P.W. Van Ophem, W.N. Reijnders, R.J. Van Spanning, J.A. Duine, A.H. Stouthamer, and N. Harms. 1995. Isolation, sequencing, and mutagenesis of the gene encoding NAD- and glutathione-dependent formaldehyde dehydrogenase (GD-FALDH) from Paracoccus denitrificans, in which GDFALDH is essential for methylotrophic growth. J. Bacteriol. 177:247-251.

14.Sambrook, J., E.F. Fritsch, and T. Maniatis. 1989. Molecular Cloning: A Laboratory Manual, 2nd ed. CSH Laboratory Press, Cold Spring Harbor, NY.

15.Simon, R., U. Priefer, and A. Puhler. 1983. A broad host range mobilization system for in vivo genetic engineering: transposon mutagenesis in Gram-negative bacteria. Bio/Technology 1:784-791.

16.Tatusov, R.L., M.Y. Galperin, D.A. Natale, and E.V. Koonin. 2000. The COG database: a tool for genome-scale analysis of protein functions and evolution. Nucleic Acids Res. 28:33-36.

17.Vieira, J. and J. Messing. 1982. The pUC plasmids, an M13mp7-derived system for insertion mutagenesis and sequencing with synthetic universal primers. Gene 19:259-268.

18.Vorholt, J.A., C.J. Marx, M.E. Lidstrom, and R.K. Thauer. 2000. Novel formaldehyde-activating enzyme in Methylobacterium extorquens AM1 required for growth on methanol. J. Bacteriol. 182:6645-6650.

19.Wild, J., M. Sektas, Z. Hradecna, and W. Szybalski. 1998. Targeting and retrofitting pre-existing libraries of transposon insertions with FRT and oriV elements for in-vivo generation of large quantities of any genomic fragment. Gene 223:55-66.

Received 16 April 2002; accepted 17 July 2002.

Address correspondence to:

Dr. Mary E. Lidstrom

Department of Chemical Engineering

University of Washington

Box 351750

Seattle, WA 98195-1750, USA

e-mail: lidstrom@u.washington.edu

For reprints of this or any other article, contact Reprints@BioTechniques.com 